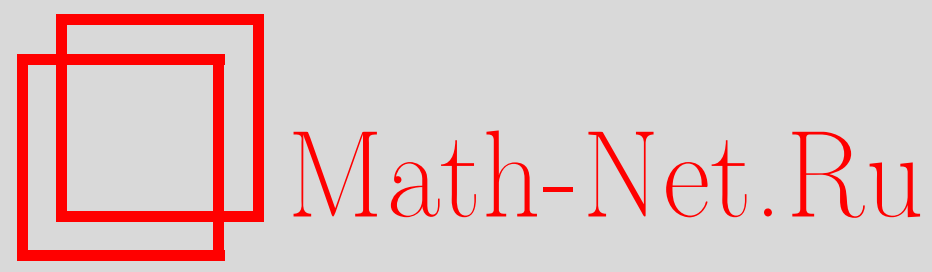

В. А. Копытцев, В. Г. Михайлов, Теоремы пуассоновского типа для числа специальных решений случайного линейного включения, Дискрет. матем., 2010, том 22, выпуск 2, 321

DOI: https://doi.org/10.4213/dm1091

Использование Общероссийского математического портала Math-Net.Ru подразумевает, что вы прочитали и согласны с пользовательским соглашением http://www . mathnet.ru/rus/agreement

Параметры загрузки:

IP : 3.93.64.190

26 апреля 2023 г., 12:43:28 


\title{
Теоремы пуассоновского типа для числа специальных решений случайного линейного Включения
}

\author{
() 2010 г. В. А. Копытцев, В. Г. Михайлов
}

\begin{abstract}
При заданных множествах $D$ и $B$ векторов линейных пространств над конечным полем размерности $n$ и $T$ соответственно и случайной матрице $A$ размера $T \times n$ над этим полем рассматривается распределение числа векторов, удовлетворяющих системе соотношений $x \in D, A x \in B$ (числа решений случайного линейного включения $A x \in B$, принадлежащих множеству $D$ ). Указаны условия, обеспечивающие при $n, T \rightarrow \infty$ сходимость этого распределения к простому и к сложному распределениям Пуассона. В них предполагается, что распределение матрицы $A$ сближается с равномерным распределением, а хотя бы одно из множеств $D$ или $B$ удовлетворяет условию, которое в работе названо условием асимптотической свободы от линейных комбинаций. Эти результаты обобщают известные предельные теоремы о числе специальных решений систем случайных линейных уравнений. Они, в частности, позволяют описать асимптотическое поведение числа приближенных решений заведомо совместных систем.

Работа выполнена при частичной поддержке Российского фонда фундаментальных исследований (проект 08-01-00078a).
\end{abstract}

\section{1. Введение}

Линейным включением (над полем $K$ ) размерности $T$ относительно $n$-мерного вектора мы называем запись $A x \in B$, где $A$ - матрица из элементов поля $K$ размера $T$ на $n$, a $B$ - некоторое множество $T$-мерных векторов над этим полем. Решением линейного включения будет любой вектор $x n$-мерного линейного пространства $V^{n}$ над полем $K$, для которого вектор $A x$ принадлежит множеству $B$. Полным решением линейного включения является множество всех таких векторов $x$.

К линейным включениям приводит, например, задача о числе приближенных решений заведомо совместной системы линейных уравнений $A x=A x^{0}$. Под приближенными решениями понимаются такие векторы $x$, для которых разности $x-x^{0}$ и $A x-A x^{0}$ малы в том или ином смысле. Например, пусть они имеют относительно небольшое число ненулевых координат (их число у вектора $y$ далее обозначаем как $\|y\|$ ). Преобразуем нашу систему заменой $x-x^{0}=y$ в систему однородных уравнений $A y=0$. Тогда мы придем к задаче о числе тех решений линейного включения $A y \in B_{r}$, где

$$
B_{r}=\{b:\|b\| \leqslant r\}
$$


которые принадлежат множеству

$$
D_{r}=\{y: 1 \leqslant\|y\| \leqslant r\} .
$$

Далее решения линейного включения, принадлежащие заданному собственному подмножеству $D$, будем называть специальными.

В настоящей работе изучаются свойства распределения числа специальных решений случайного линейного включения над полем $K=G F(q)$ со случайной матрицей $A=\left\|a_{i, j}\right\|$, элементы которой независимы в совокупности и распределены с вероятностями

$$
\mathbf{P}\left\{a_{i, j}=k\right\}=\frac{1+\Delta_{i, j}(k)}{q}, \quad k \in K,
$$

где

$$
\sum_{k \in K} \Delta_{i, j}(k)=0, \quad i=1, \ldots, T, \quad j=1, \ldots, n .
$$

Пусть

$$
\Delta=\max _{i, j, k}\left|\Delta_{i, j}(k)\right|<1 .
$$

Рассматривается случай, когда параметры $n$ и $T$ согласованно возрастают: $n, T \rightarrow \infty$. Нас интересуют условия, которым должны удовлетворять множества $B$ и $D$ для того, чтобы распределение числа специальных решений случайного линейного включения сходилось к пуассоновскому или сложному пуассоновскому распределению. Аналогичные вопросы для числа всех решений случайного линейного включения изучались в рабо$\operatorname{Tax}[4,5]$.

В работе [4] были получены достаточные условия сходимости распределения числа ненулевых решений случайного линейного включения над полем $G F(q)$ к сложному пуассоновскому распределению. В ней было использовано следующее условие на правую часть включения.

Пусть $N\left(a_{1}, a_{2}, a_{3}, d, B\right)$ обозначает число решений системы из $T$ линейных уравнений над полем $K$, записанной в виде уравнения

$$
a_{1} u^{1} \oplus a_{2} u^{2} \oplus a_{3} u^{3}=d,
$$

относительно тройки векторов $\left(u^{1}, u^{2}, u^{3}\right) \in B^{3}$, где $B-$ некоторое заданное подмножество $V^{T}, a_{1}, a_{2}, a_{3} \in K \backslash\{0\}, d \in V^{T}$. Положим

$$
\begin{aligned}
N(B) & =\max _{a_{1}, a_{2}, a_{3}, d} N\left(a_{1}, a_{2}, a_{3}, d, B\right), \\
\rho(B) & =\frac{N(B)}{|B|^{2}},
\end{aligned}
$$

где $|B|$ обозначает число элементов конечного множества $B$. Очевидно, что $0 \leqslant \rho(B) \leqslant 1$.

Отношение $\rho(B)=N(B) /|B|^{2}$ является своего рода мерой отличия множества $B$ от линейного или аффинного пространства. Для линейных и аффинных подпространств эта мера принимает максимально возможное значение $\rho(B)=1$ (см. [5]). Если $0<\rho(B)<1$, то множество $B$ можно считать частично линейным. Условие

$$
\rho(B) \rightarrow 0
$$

можно трактовать как асимптотическую нелинейность множества $B=B(T)$ при $T \rightarrow \infty$, или, что более точно (см. раздел 5), как его асимптотическую свободу от линейных комбинаций. 
Замечание 1. Непосредственно из определения величины $\rho(B)$ вытекают следующие свойства.

(А) Соотношения $\rho\left(B \oplus b^{0}\right) \rightarrow 0$ выполнены (или не выполнены) одновременно при всех $b^{0}=b^{0}(T) \in V^{T}$.

(Б) Из соотношения $\rho(B) \rightarrow 0$ вытекает соотношение $\rho\left(B \cup B^{\prime}\right) \rightarrow 0$ для любого множества $B^{\prime}$, удовлетворяющего условию $\left|B^{\prime}\right| /|B| \rightarrow 0$ при $T \rightarrow \infty$.

Теорема 1. Пусть

$$
T \rightarrow \infty, \quad r \geqslant 1, \quad r T^{-1} \leqslant \rho
$$

при некотором $0<\rho<(q-1) / q$. Тогда для множеств

$$
\begin{aligned}
& S_{r}\left(b^{0}\right)=\left\{b \in V^{T}: 1 \leqslant\left\|b-b^{0}\right\| \leqslant r\right\}, \\
& S_{r}^{\prime}\left(b^{0}\right)=\left\{b \in V^{T}:\left\|b-b^{0}\right\|=r\right\}
\end{aligned}
$$

при любых $b^{0}=b^{0}(T) \in V^{T}$ выполнено соотномение (3).

Обозначим через $\xi=\xi(D, A, B)$ число тех ненулевых решений включения $A x \in B$, которые принадлежат множеству $D$. Другими словами,

$$
\xi=\xi(D, A, B)=|\{x \in D, A x \in B\}| .
$$

Цель работы - исследовать предельное поведение этой случайной величины при таком изменении параметров, когда $|D| \rightarrow \infty$ и

$$
\mathbf{E} \xi(D, A, B)=q^{-T}|D \times B| \rightarrow \lambda, \quad 0<\lambda<\infty .
$$

Основные результаты статьи сформулированы в двух следующих разделах. В разделе 2 описано совместное распределение чисел специальных решений $\xi\left(D, A, B_{k}\right)$ случайных линейных включений $A x \in B_{k}, k=1, \ldots, s$, с общей левой частью и с непересекающимися множествами $B_{1}, \ldots, B_{s}$ в правых частях включений в том случае, когда в множестве $D \times B=D \times \bigcup_{k=1}^{s} B_{k}$ нет подобных векторов или их относительно мало. Напомним, что подобными называются векторы, получаемые друг из друга умножением на ненулевой элемент поля. Выведены условия выполнения для величин $\xi\left(D, A, B_{k}\right)$ многомерной предельной теоремы Пуассона (теорема 2). В разделе 3 рассмотрен случай, когда $s=1$, а подобные векторы в множестве $B=B_{1}$ имеются, причем в числе, сравнимом по порядку с $|D \times B|$. Тогда предельное распределение величины $\xi(D, A, B)$ оказывается сложным пуассоновским распределением (теорема 3). Раздел 4 посвящен доказательству теорем 2 и 3. Теорема 1 доказывается в разделе 5 .

\section{2. Многомерная предельная теорема Пуассона}

Зададим множество $D=D(n) \subset V^{n}$ и набор попарно не пересекающихся множеств $B_{1}=B_{1}(T), \ldots, B_{S}=B_{S}(T) \subset V^{T}$. Положим $B=B_{1} \cup \ldots \cup B_{S}$.

Теорема 2. Пусть $K=G F(q)$, распределение элементов матрищы А описывается формулой (1), $0 \notin D$, множество $D \times B$ не содержит подобных векторов и при $n, T \rightarrow \infty$ выполнены соотношения $T \Delta \rightarrow 0,|D| \rightarrow \infty$,

$$
\begin{aligned}
q^{-T}\left|D \| B_{k}\right| & \rightarrow \lambda_{k}, \quad 0 \leqslant \lambda_{k}<\infty, \quad k=1, \ldots, s, \quad \exists k: \lambda_{k}>0, \\
\rho(D) \rho(B) & \rightarrow 0 .
\end{aligned}
$$


Тогда случайные величины $\xi\left(D, A, B_{k}\right)$ асимптотически независимы, а их распределения сходятся к распределениям Пуассона с параметрами $\lambda_{k}$ соответственно.

Замечание 2. В теореме 2 и далее считаем, что вырожденное в нуле распределение является распределением Пуассона с параметром $\lambda=0$.

Замечание 3. Условие (8) определяет значения параметров предельных распределений случайных величин $\xi\left(D, A, B_{k}\right)$, а условие (9) означает, что по крайней мере для одного из множеств $D$ и $B$ выполнено соотношение (3).

Замечание 4. Условие (9) в теоремах для числа решений случайных линейных включений используется впервые. Если при переходе к пределу $|B| \leqslant C<\infty$, то соотношение $\rho(B) \rightarrow 0$ не может выполняться. В этом случае условие (9) принимает вид $\rho(D) \rightarrow 0$.

Замечание 5. Если $0 \in B$, то условие отсутствия подобных векторов в множестве $D \times B$ эквивалентно отсутствию подобных векторов в множестве $D$.

Замечание 6. Условия отсутствия подобных векторов в множествах $D, B$ и $D \times B$ выполнены автоматически, если $K=G F(2)$.

Замечание 7. В случае, когда $B_{1}=\left\{b^{1}\right\}, \ldots, B_{s}=\left\{b^{s}\right\}$, теорема 2 рассматривает поведение совместного распределения чисел специальных решений у $s$ случайных систем линейных уравнений $A x=b^{1}, \ldots, A x=b^{s}$ с одинаковой левой и разными правыми частями. Для этого случая из нее вытекает следующее утверждение.

Следствие 1. Пусть $K=G F(q)$, распределение элементов матрицы $A$ описывается формулой (1), $0 \notin D, b^{1}, \ldots, b^{s} \in V^{T} \backslash\{0\}$ и среди них нет подобных векторов. Если $n, T \rightarrow \infty$, выполнены соотношения $T \Delta \rightarrow 0, \rho(D) \rightarrow 0, u$

$$
q^{-T}|D| \rightarrow \lambda, \quad 0<\lambda<\infty,
$$

то числа тех решений систем $A x=b^{1}, \ldots, A x=b^{s}$, которые принадлежат множеству $D$, асимптотически независимы, а распределение каждого из них сходится $к$ распределению Пуассона с параметром $\lambda$.

Замечание 8. Если в множестве $D$ нет подобных векторов, то утверждение следствия 1 распространяется также на систему $A x=0$.

Замечание 9. Сформулированные выше результаты естественным образом распространяются на случай, когда множество $D \times B$ (либо множество $D$ в случае замечания 8) содержит подобные векторы, но их общее число бесконечно мало по сравнению с $|D \times B|$ при $n, T \rightarrow \infty$.

\section{3. Теорема о сходимости к сложному пуассоновскому распределению}

Опишем теперь асимптотическое поведение распределения числа решений, принадлежащих множеству $D$, у случайного линейного включения $A x \in B$ в том случае, когда множество $D \times B$ содержит подобные векторы в числе, сравнимом с $|D \times B|$. Разобьем множество $D \times B$ на классы $D B_{1}, \ldots, D B_{M}$ подобных векторов, где $M$ - общее число таких классов. Положим

$$
l_{r}(D, B)=\left|\left\{k \in\{1, \ldots, M\}:\left|D B_{k}\right|=r\right\}\right|, \quad r=1, \ldots, q-1 .
$$


Теорема 3. Пусть $K=G F(q)$, распределение элементов матрищы А описывается формулой (1), $0 \notin D$, а при $n, T \rightarrow \infty$ выполнены соотношения $T \Delta \rightarrow 0,|D| \rightarrow \infty$, (9) $u$ условие

$$
q^{-T} l_{r}(D, B) \rightarrow \lambda_{r}, \quad 0 \leqslant \lambda_{r}<\infty, \quad r=1, \ldots, q-1, \quad \exists r: \lambda_{r}>0 .
$$

Тогда распределение случайной величины $\xi(D, A, B)$ сходится к распределению выражения $\pi_{1}+2 \pi_{2}+\ldots+(q-1) \pi_{q-1}$, где $\pi_{1}, \ldots, \pi_{q-1}-$ независимые в совокупности случайные величины, распределенные по закону Пуассона с параметрами $\lambda_{1}, \ldots, \lambda_{q-1}$ соответственно.

Рассмотрим частный случай, когда оба множества $D$ и $B$ замкнуты относительно умножения векторов на ненулевые элементы поля. Из теоремы 3 вытекает следующее утверждение.

Следствие 2. Пусть $K=G F(q)$, распределение элементов матрищы $A$ описывается формулой (1), $0 \notin D$, множества $D$ и $B$ замкнуты относительно умножения векторов на ненулевые элементы поля $K, n, T \rightarrow \infty, T \Delta \rightarrow 0,|D| \rightarrow \infty$, и выполнены условия (9) $u$ (7). Тогда распределение случайной величинь $\xi(D, A, B) /(q-1)$ сходится к распределению Пуассона с параметром $\lambda /(q-1)$.

Замечание 10. В случае, когда

$$
\begin{aligned}
& D=D_{r}=\left\{x \in V^{n}: 1 \leqslant\|x\| \leqslant r\right\}, \\
& B=B_{r}=\left\{b \in V^{T}:\|b\| \leqslant r\right\},
\end{aligned}
$$

следствие 2 описывает предельное поведение числа приближенных решений заведомо совместной системы линейных уравнений $A x=A x^{0}$ (см. раздел 1). В этом случае с учетом теоремы 1 и замечания 1 получаем следующие утверждения.

Следствие 3. Пусть $K=G F(q)$, распределение элементов матрищы $A$ описывается формулой (1), $n, T \rightarrow \infty, T \Delta \rightarrow 0$, параметры $r_{1}, r_{2} \geqslant 1$ меняются так, что

$$
q^{-T} \sum_{i=1}^{r_{1}}\left(\begin{array}{l}
n \\
i
\end{array}\right)(q-1)^{i} \sum_{j=1}^{r_{2}}\left(\begin{array}{l}
T \\
j
\end{array}\right)(q-1)^{j} \rightarrow \lambda, \quad 0<\lambda<\infty,
$$

и при некотором $\rho, 0<\rho<(q-1) / q$, выполнено хотя бы одно из условий $r_{1} n^{-1} \leqslant \rho$, $r_{2} T^{-1} \leqslant \rho$. Тогда распределение случайной величины $\xi_{r_{1}, r_{2}}\left(x^{0}\right) /(q-1)$, где $\xi_{r_{1}, r_{2}}\left(x^{0}\right)-$ число тех $x \in V^{n}$, для которых $1 \leqslant\left\|x-x^{0}\right\| \leqslant r_{1} u\left\|A x-A x^{0}\right\| \leqslant r_{2}$, при любом изменении $x^{0}$ сходится к распределению Пуассона с параметром $\lambda /(q-1)$.

Следствие 4. Пусть $K=G F(q)$, распределение элементов матрицы $A$ определяется соотношениями (1), $n, T \rightarrow \infty, T \Delta \rightarrow 0$, параметр $r \geqslant 1$ меняется так, что

$$
q^{-T} \sum_{i=1}^{r}\left(\begin{array}{l}
n \\
i
\end{array}\right)(q-1)^{i} \rightarrow \lambda, \quad 0<\lambda<\infty,
$$

и при некотором $\rho, 0<\rho<(q-1) / q$, выполнено условие $r n^{-1} \leqslant \rho$. Тогда распределение случайной величины $\xi_{r}\left(x^{0}\right) /(q-1)$, где $\xi_{r}\left(x^{0}\right)-$ число тех решений системь уравнений $A x=A x^{0}$, для которых $1 \leqslant\left\|x-x^{0}\right\| \leqslant r$, при любом изменении вектора $x^{0}$ сходится $\kappa$ распределению Пуассона с параметром $\lambda /(q-1)$. 
Замечание 11. Следствия 3 и 4 существенно дополняют результаты работ [1] и [2], распространяя некоторые из них на случай множеств общего вида.

Значительный интерес теорема 3 представляет в случае, когда $B=\left\{0^{T}\right\}$. Тогда речь идет о числе специальных решений системы случайных однородных уравнений $A x=0^{T}$. Положим $l_{r}(D)=l_{r}\left(D,\left\{0^{T}\right\}\right)$.

Следствие 5. Пусть $K=G F(q)$, распределение элементов матрищы $A$ описывается формулой (1), $0^{n} \notin D$, а при $n, T \rightarrow \infty$ выполнены соотношения $T \Delta \rightarrow 0,|D| \rightarrow \infty$, $\rho(D) \rightarrow 0$ и условие

$$
q^{-T} l_{r}(D) \rightarrow \lambda_{r}, \quad 0 \leqslant \lambda_{r}<\infty, \quad r=1, \ldots, q-1, \quad \exists r: \lambda_{r}>0 .
$$

Тогда распределение случайной величины $\xi\left(D, A,\left\{0^{T}\right\}\right)$ сходится к распределению выражения $\pi_{1}+2 \pi_{2}+\ldots+(q-1) \pi_{q-1}$, где $\pi_{1}, \ldots, \pi_{q-1}-$ независимые в совокупности случайные величины, распределенные по закону Пуассона с параметрами $\lambda_{1}, \ldots, \lambda_{q-1}$ соответственно.

Замечание 12. Предельная теорема для числа специальных решений системы случайных однородных уравнений $A x=0$ при более жестких предположениях относительно свойств распределения элементов матрицы $A$ была доказана в [5]. Там предполагалось, что $\Delta=0$.

\section{4. Доказательство теорем 2 и 3}

Введем характеристику, которой мы будем описывать асимптотические свойства множества $D \subseteq V^{n}$. Положим

$$
\bar{D}_{k, j}=\left\{\left(x^{1}, \ldots, x^{k}\right) \in D^{k}: x^{\alpha} \neq c x^{\beta}, c \in K, \alpha \neq \beta, \operatorname{rank}\left(x^{1}, \ldots, x^{k}\right)=j\right\} .
$$

Пусть $I\{E\}$ обозначает индикатор случайного события $E$. Рассмотрим систему случайных индикаторов $\{I\{A x=b\}:(x, b) \in J\}$, где $J \subseteq D \times B$. Пусть задан набор $R_{1} \ldots, R_{s}$ непересекающихся подмножеств множества $J$. Пусть

$$
\xi_{u}=\sum_{(x, b) \in R_{u}} I\{A x=b\}, \quad u=1, \ldots, s .
$$

Теорема 4. Пусть $K=G F(q)$, распределение элементов матрищы А описывается формулой (1), $0^{n} \notin D$, множество $J$ не содержит подобных векторов и при $n, T \rightarrow \infty$ выполнены соотношения $T \Delta \rightarrow 0,|D| \rightarrow \infty$,

$$
\begin{aligned}
q^{-T}|D||B| & =O(1), \\
q^{-T}\left|R_{k}\right| & \rightarrow \lambda_{k}^{\prime}, \quad 0 \leqslant \lambda_{k}^{\prime}<\infty, \quad k=1, \ldots, s,
\end{aligned}
$$

и при всех $k>j \geqslant 2$

$$
\frac{\left|\bar{D}_{k, j}\right|}{|D|^{j}} \rho(B) \rightarrow 0 .
$$

Тогда случайные величины $\xi_{1}, \ldots, \xi_{\text {s }}$ асимптотически независимы, а их распределения сходятся к распределениям Пуассона с параметрами $\lambda_{1}, \ldots, \lambda_{s}$ соответственно. 
Для доказательства теоремы 4 мы воспользуемся многомерной версией известной теоремы Б. А. Севастьянова об условиях справедливости предельной теоремы Пуассона для сумм зависимых индикаторов (см. [3]). Ее условия используют понятие исключительных множеств, к заданию которых мы и приступаем. Пусть (используем обозначение $\left.v^{i}=\left(x^{i}, b^{i}\right)\right)$

$$
\begin{aligned}
J_{k} & =\left\{\left(v^{1}, \ldots, v^{k}\right) \in J^{k}: v^{\alpha} \neq v^{\beta}(\alpha \neq \beta)\right\}, \\
D_{k, j} & =\left\{\left(x^{1}, \ldots, x^{k}\right) \in D^{k}: \operatorname{rank}\left(x^{1}, \ldots, x^{k}\right)=j\right\}, \\
D_{k} & =\bigcup_{j=1}^{k-1} D_{k, j} .
\end{aligned}
$$

Нетрудно убедиться, что

$$
\left|D_{k, j}\right| \leqslant\left(\begin{array}{c}
k \\
j
\end{array}\right) q^{j(k-j)}|D|^{j} .
$$

Исключительные множества $I_{k} \subset J_{k}$ определим равенством

$$
I_{k}=\left\{\left(\left(x^{1}, b^{1}\right), \ldots,\left(x^{k}, b^{k}\right)\right) \in J_{k}:\left(x^{1}, \ldots, x^{k}\right) \in D_{k}\right\} .
$$

Утверждение теоремы 4 будет доказано, если убедиться, что выполнены условия

$$
\begin{aligned}
& \sum_{(x, b) \in R_{u}} \mathbf{P}\{A x=b\} \rightarrow \lambda_{u}^{\prime}, \quad u=1, \ldots, s, \\
& \max _{(x, b) \in J} \mathbf{P}\{A x=b\} \rightarrow 0
\end{aligned}
$$

и при всех $k=2,3, \ldots$ (используем обозначение $\left.v^{i}=\left(x^{i}, b^{i}\right)\right)$

$$
\begin{array}{r}
\max _{\left(v^{1}, \ldots, v^{k}\right) \in J_{k} \backslash I_{k}}\left|\frac{\mathbf{P}\left\{A x^{1}=b^{1}, \ldots, A x^{k}=b^{k}\right\}}{\mathbf{P}\left\{A x^{1}=b^{1}\right\} \ldots \mathbf{P}\left\{A x^{k}=b^{k}\right\}}-1\right| \rightarrow 0, \\
\sum_{\left(v^{1}, \ldots, v^{k}\right) \in I_{k}} \prod_{j=1}^{k} \mathbf{P}\left\{A x^{j}=b^{j}\right\} \rightarrow 0, \\
\sum_{\left(v^{1}, \ldots, v^{k}\right) \in I_{k}} \mathbf{P}\left\{A x^{1}=b^{1}, \ldots, A x^{k}=b^{k}\right\} \rightarrow 0 .
\end{array}
$$

Приступим к проверке этих условий. Нам понадобится следующая лемма.

Лемма 1. Пусть $x^{1}, \ldots, x^{k} \in D u b^{1}, \ldots, b^{k} \in V^{T}$. Тогда при всех $k=1,2, \ldots$

$$
\mathbf{P}\left\{A x^{1}=b^{1}, \ldots, A x^{k}=b^{k}\right\} \leqslant\left(\frac{1+\Delta}{q}\right)^{l T},
$$

если $\operatorname{rank}\left(x^{1}, \ldots, x^{k}\right)=l \leqslant k-1, u$

$$
\left(\frac{1-\Delta}{q}\right)^{k T} \leqslant \mathbf{P}\left\{A x^{1}=b^{1}, \ldots, A x^{k}=b^{k}\right\} \leqslant\left(\frac{1+\Delta}{q}\right)^{k T},
$$

если $\operatorname{rank}\left(x^{1}, \ldots, x^{k}\right)=k$. 
Доказательство леммы 1 аналогично доказательству леммы 3 в [2]. Поэтому мы его не приводим.

Из неравенств (24), условий $T \Delta \rightarrow 0$ и (10) следуют соотношения (18) и (19).

Проверим выполнение условия (20). Согласно определениям, множество $J_{k} \backslash I_{k}$ образуют все наборы $\left(\left(x^{1}, b^{1}\right), \ldots,\left(x^{k}, b^{k}\right)\right)$, в которых $\left(x^{1}, \ldots, x^{k}\right) \in D_{k, k}$. Из неравенств (24) вытекает, что для них

$$
\left(\frac{1-\Delta}{1+\Delta}\right)^{k T} \leqslant \frac{\mathbf{P}\left\{A x^{1}=b^{1}, \ldots, A x^{k}=b^{k}\right\}}{\mathbf{P}\left\{A x^{1}=b^{1}\right\} \ldots \mathbf{P}\left\{A x^{k}=b^{k}\right\}} \leqslant\left(\frac{1+\Delta}{1-\Delta}\right)^{k T} .
$$

Поэтому из условия $T \Delta \rightarrow 0$ следует (20).

Проверим соотношение (21) при $k \geqslant 2$. Используя условия (24) (где $k=1$ ) и (17), получаем, что

$$
\begin{aligned}
\sum_{\left(v^{1}, \ldots, v^{k}\right) \in I_{k}} \prod_{j=1}^{k} \mathbf{P}\left\{A x^{j}=b^{j}\right\} & \leqslant \sum_{\left(x^{1}, \ldots, x^{k}\right) \in D_{k}} \sum_{b^{1} \in B} \ldots \sum_{b^{k} \in B} \prod_{j=1}^{k} \mathbf{P}\left\{A x^{j}=b^{j}\right\} \\
& =\sum_{\left(x^{1}, \ldots, x^{k}\right) \in D_{k}} \prod_{i=1}^{k} \mathbf{P}\left\{A x^{i} \in B\right\} \\
& =\sum_{j=1}^{k-1} \sum_{\left(x^{1}, \ldots, x^{k}\right) \in D_{k, j}} \prod_{i=1}^{k} \mathbf{P}\left\{A x^{i} \in B\right\} \\
& \leqslant \sum_{j=1}^{k-1} \sum_{\left(x^{1}, \ldots, x^{k}\right) \in D_{k, j}}\left(\frac{1+\Delta}{q}\right)^{k T}|B|^{k} \\
& =\sum_{j=1}^{k-1}\left|D_{k, j}\right|\left(\frac{1+\Delta}{q}\right)^{k T}|B|^{k} \\
& \leqslant\left(\frac{|D||B|}{q^{T}}\right)^{k}(1+\Delta)^{k T} \frac{1}{|D|^{k}} \sum_{j=1}^{k-1}\left(\begin{array}{l}
k \\
j
\end{array}\right) q^{j(k-j)}|D|^{j} .
\end{aligned}
$$

Из условий (14), $T \Delta \rightarrow 0$ и $|\Delta| \rightarrow \infty$ следует, что выражение в правой части (25) стремится к нулю при всех $k \geqslant 2$. Соотношение (21) доказано.

Наконец, проверим соотношение (22). Пусть опять $k \geqslant 2$. Из определений следует равенство

$$
\begin{aligned}
\sum_{\left(v^{1}, \ldots, v^{k}\right) \in I_{k}} \mathbf{P}\left\{A x^{1}=b^{1}, \ldots, A x^{k}=b^{k}\right\} & \sum_{\left(v^{1}, \ldots, v^{k}\right) \in I_{k},\left(x^{1}, \ldots, x^{k}\right) \in D_{k}} \mathbf{P}\left\{A x^{1}=b^{1}, \ldots, A x^{k}=b^{k}\right\} .
\end{aligned}
$$

Заметим, что $\bar{D}_{k, 1}=\varnothing, k=2,3, \ldots$, и положим

$$
\bar{D}_{k}=\bigcup_{j=2}^{k-1} \bar{D}_{k, j}
$$


Лемма 2. Пусть $D \times B$ не содержит подобных векторов, $\left(x^{1}, \ldots, x^{k}\right) \in D_{k} \backslash \bar{D}_{k}$, $\left(\left(x^{1}, b^{1}\right), \ldots,\left(x^{k}, b^{k}\right)\right) \in J_{k}$. Тогда

$$
\mathbf{P}\left\{A x^{1}=b^{1}, \ldots, A x^{k}=b^{k}\right\}=0 .
$$

Доказательство. Предположим противное, а именно, предположим, что для некоторого набора векторов $\left(x^{1}, \ldots, x^{k}\right) \in D_{k} \backslash \bar{D}_{k}$ выполнено неравенство

$$
\mathbf{P}\left\{A x^{1}=b^{1}, \ldots, A x^{k}=b^{k}\right\}>0 .
$$

Это означает, что для некоторой реализации $A^{\prime}$ случайной матрицы $A$ выполнен набор из $k$ равенств $A^{\prime} x^{1}=b^{1}, \ldots, A^{\prime} x^{k}=b^{k}$. Согласно определениям, в нашем наборе $\left(x^{1}, \ldots, x^{k}\right) \in D_{k} \backslash \bar{D}_{k}$ найдутся такие векторы $x^{i} \in D, x^{j} \in D, x^{i} \neq x^{j}$, и найдется такое число $\alpha \in K \backslash\{0\}$, что $x^{j}=\alpha x^{j}$.

Пусть $\alpha=1$. Тогда из равенства $x^{j}=x^{i}$ следует, что и $b^{j}=b^{i}$. Последнее противоречит условию $\left(\left(x^{1}, b^{1}\right), \ldots,\left(x^{k}, b^{k}\right)\right) \in J_{k}$. Значит, наше предположение ошибочно.

Пусть $q \geqslant 3$ и $\alpha \neq 1$. Тогда $\left(x^{i}, b^{i}\right) \in D \times B,\left(x^{j}, b^{j}\right)=\alpha\left(x^{i}, b^{i}\right) \in D \times B$. Это противоречит условию, что множество $D \times B$ не содержит подобных векторов. Таким образом, и в этом случае наше предположение ошибочно. Значит, $\mathbf{P}\left\{A x^{1}=b^{1}, \ldots, A x^{k}=b^{k}\right\}=0$. Лемма доказана.

Из (26) и леммы 2 следует, что

$$
\begin{gathered}
\sum_{\left(v^{1}, \ldots, v^{k}\right) \in I_{k}} \mathbf{P}\left\{A x^{1}=b^{1}, \ldots, A x^{k}=b^{k}\right\} \\
=\sum_{\left(v^{1}, \ldots, v^{k}\right) \in I_{k},\left(x^{1}, \ldots, x^{k}\right) \in \bar{D}_{k}} \mathbf{P}\left\{A x^{1}=b^{1}, \ldots, A x^{k}=b^{k}\right\} .
\end{gathered}
$$

Теперь рассмотрим произвольный набор $x^{1}, \ldots, x^{r}$ векторов пространства $V^{n}$. Ему соответствует система $L_{x}\left(x^{1}, \ldots, x^{r}\right)$ однородных линейных уравнений вида

$$
\alpha_{1} x^{1} \oplus \ldots \oplus \alpha_{r} x^{r}=0
$$

возможно пустая, состоящая из всех линейных соотношений, которым удовлетворяет набор $x^{1}, \ldots, x^{r}$. Системе $L_{x}\left(x^{1}, \ldots, x^{r}\right)$ сопоставим идентичную по составу систему $L_{b}\left(x^{1}, \ldots, x^{r}\right)$ из линейных уравнений относительно $b^{1}, \ldots, b^{r} \in B$. Обозначим через $B\left(x^{1}, \ldots, x^{r}\right)$ множество решений $\left(b^{1}, \ldots, b^{r}\right), b^{1}, \ldots, b^{r} \in B$, системы $L_{b}\left(x^{1}, \ldots, x^{r}\right)$.

Лемма 3. Пусть $\left(b^{1}, \ldots, b^{r}\right) \notin B\left(x^{1}, \ldots, x^{r}\right),\left(x^{1}, \ldots, x^{r}\right) \in \bar{D}_{k}$. Тогда

$$
\mathbf{P}\left\{A x^{1}=b^{1}, \ldots, A x^{k}=b^{k}\right\}=0 .
$$

Доказательство. Доказательство леммы 3 проведем аналогично доказательству леммы 2. Предположим противное, именно, что для некоторых наборов векторов $\left(x^{1}, \ldots, x^{r}\right) \in \bar{D}_{k}$ и $\left(b^{1}, \ldots, b^{r}\right) \notin B\left(x^{1}, \ldots, x^{r}\right)$ выполнено неравенство

$$
\mathbf{P}\left\{A x^{1}=b^{1}, \ldots, A x^{k}=b^{k}\right\}>0 .
$$

Тогда найдется такой набор элементов поля $\left(\alpha_{1}, \ldots, \alpha_{k}\right) \neq(0, \ldots, 0)$, что

$$
\alpha_{1} x^{1} \oplus \ldots \oplus \alpha_{k} x^{k}=0, \quad \alpha_{1} b^{1} \oplus \ldots \oplus \alpha_{k} b^{k} \neq 0,
$$


и, кроме того, для некоторой реализации $A^{\prime}$ случайной матрицы $A$ будет выполнен набор равенств

$$
A^{\prime} x^{1}=b^{1}, \quad \ldots, \quad A^{\prime} x^{k}=b^{k} .
$$

Очевидно, что условия (28) и (29) не могут выполняться одновременно. Следовательно, $\mathbf{P}\left\{A x^{1}=b^{1}, \ldots, A x^{k}=b^{k}\right\}=0$. Лемма 3 доказана.

Из леммы 3 следует, что

$$
\begin{aligned}
& \sum_{\substack{\left(v^{1}, \ldots, v^{k}\right) \in I_{k} \\
\left(x^{1}, \ldots, x^{k}\right) \in \bar{D}_{k}}} \mathbf{P}\left\{A x^{1}=b^{1}, \ldots, A x^{k}=b^{k}\right\} \\
& \quad=\sum_{j=2}^{k-1} \sum_{\left(x^{1}, \ldots, x^{k}\right) \in \bar{D}_{k, j}} \sum_{\left(b^{1}, \ldots, b^{r}\right) \in B\left(x^{1}, \ldots, x^{r}\right)} \mathbf{P}\left\{A x^{1}=b^{1}, \ldots, A x^{k}=b^{k}\right\} .
\end{aligned}
$$

Теперь нам понадобится одно вспомогательное утверждение, связанное с характеристикой $\rho(B)$ множества $B$.

Лемма 4. Пусть $2 \leqslant j \leqslant k-1 u\left(x^{1}, \ldots, x^{k}\right) \in \bar{D}_{k}$. Тогда

$$
\left|B\left(x^{1}, \ldots, x^{r}\right)\right| \leqslant \rho(B)|B|^{j} .
$$

Доказательство. Так как $\operatorname{rank}\left(x^{1}, \ldots, x^{r}\right) \leqslant k-1$, система $L_{b}\left(x^{1}, \ldots, x^{r}\right)$ непуста. Перенумеруем векторы $x^{1}, \ldots, x^{r}$ так, чтобы первые $j$ из них составляли базис. Преобразованная система $L_{b}\left(x^{1}, \ldots, x^{r}\right)$ будет содержать уравнение вида $b^{j+1}=l_{j+1}\left(b^{1}, \ldots, b^{j}\right)$, где $l_{j+1}$ - однородная линейная функция своих аргументов. При этом значения векторов $b^{j+1}, \ldots, b^{r}$ определяются однозначно векторами $b^{1}, \ldots, b^{j}$. Поэтому величина $\left|B\left(x^{1}, \ldots, x^{r}\right)\right|$ не превосходит числа решений уравнения $b^{j+1}=l_{j+1}\left(b^{1}, \ldots, b^{j}\right)$ относительно $b^{1}, \ldots, b^{j+1}$. Так как $\left(x^{1}, \ldots, x^{k}\right) \in \bar{D}_{k}$, в системе $L_{b}\left(x^{1}, \ldots, x^{r}\right)$ нет одночленных и двучленных уравнений. Значит, уравнение $b^{j+1}=l_{j+1}\left(b^{1}, \ldots, b^{j}\right)$ содержит, по крайней мере, три существенные переменные. Не нарушая общности, можно считать, что оно имеет вид

$$
b^{j+1}=\alpha_{1} b^{1} \oplus \alpha_{2} b^{2} \oplus l_{j+1}^{\prime}\left(b^{3}, \ldots, b^{j}\right),
$$

где $l_{j+1}^{\prime}-$ некоторая линейная функция своих аргументов, возможно, тождественно равная нулю. Число решений уравнения (31) не превосходит суммы чисел решений этого уравнения относительно неизвестных $b^{1}, b^{2}, b^{j+1}$ при переборе всех значений векторов $b^{3}, \ldots, b^{j} \in B$. Следовательно, это число (и тем более, число $\left|B\left(x^{1}, \ldots, x^{r}\right)\right|$ ) не превосходит

$$
N(B)|B|^{j-2}=\rho(B)|B|^{j}
$$

(величина $N(B)$ определена во введении). Лемма 4 доказана.

Применим лемму 4 и оценку (23) к выражению в правой части цепочки неравенств 
(30), а результат подставим в (27). Получим цепочку неравенств

$$
\begin{aligned}
\sum_{\left(v^{1}, \ldots, v^{k}\right) \in I_{k}} \mathbf{P}\left\{A x^{1}=b^{1}, \ldots, A x^{k}=b^{k}\right\} & \leqslant \sum_{j=2}^{k-1} \sum_{\left(x^{1}, \ldots, x^{k}\right) \in \bar{D}_{k, j}} \sum_{\left(b^{1}, \ldots, b^{r}\right) \in B\left(x^{1}, \ldots, x^{r}\right)}\left(\frac{1+\Delta}{q}\right)^{j T} \\
& \leqslant \sum_{j=2}^{k-1} \sum_{\left(x^{1}, \ldots, x^{k}\right) \in \bar{D}_{k, j}}\left|B\left(x^{1}, \ldots, x^{r}\right)\right|\left(\frac{1+\Delta}{q}\right)^{j T} \\
& \leqslant \rho(B) \sum_{j=2}^{k-1} \sum_{\left(x^{1}, \ldots, x^{k}\right) \in \bar{D}_{k, j}}|B|^{j}\left(\frac{1+\Delta}{q}\right)^{j T} \\
& \leqslant \rho(B) \sum_{j=2}^{k-1}\left|\bar{D}_{k, j}\right||B|^{j}\left(\frac{1+\Delta}{q}\right)^{j T} \\
& \leqslant \rho(B) \sum_{j=2}^{k-1} \frac{\left|\bar{D}_{k, j}\right|}{|D|^{j}} \sum_{j=2}^{k-1}\left(\frac{(1+\Delta)^{T}|D||B|}{q}\right)^{j} .
\end{aligned}
$$

В силу условий (15), (16) и $T \Delta \rightarrow 0$, выражение в правой части (32) стремится к нулю. Значит, условие (22) тоже выполнено, и мы можем воспользоваться многомерной версией теоремы Б. А. Севастьянова. Теорема 4 доказана.

Лемма 5. Пусть $2 \leqslant j \leqslant k-1, n \rightarrow \infty$, а множество $D=D(n) \subset V^{n}$ меняется так, что $\rho(D) \rightarrow 0$. Тогда

$$
\frac{\left|\bar{D}_{k, j}\right|}{|D|^{j}} \rightarrow 0 .
$$

Из леммы 5 вытекает следующее утверждение.

Следствие 6. Пусть $n \rightarrow \infty$, множества $D=D(n) \subset V^{n} u B=B(T) \subset V^{T}$ меняются так, что $\rho(D) \rho(B) \rightarrow 0$. Тогда при всех $k>j \geqslant 2$ выполнено соотношение (16).

Доказательство леммы 5. Нетрудно убедиться, что $\bar{D}_{k, 1}=\varnothing, k=2,3, \ldots$, а в других случаях

$$
\bar{D}_{k, j}=\bigcup_{L_{k, j}} D\left(L_{k, j}\right),
$$

где $D\left(L_{k, j}\right)$ - множество наборов $\left(x^{1}, \ldots, x^{k}\right) \in D^{k}$, удовлетворяющих совокупности $L_{k, j}$ из $k-j$ линейных соотношений с не менее чем тремя ненулевыми коэффициентами в каждом (один из них можно положить равным единице), а объединение проводится по всем таким наборам. Их общее число не превосходит $\left(\sum_{l=3}^{k}(q-1)^{l-1}\left(\begin{array}{l}k \\ l\end{array}\right)\right)^{k-j}<q^{k(k-j)}$. Поэтому

$$
\left|\bar{D}_{k, j}\right|<q^{k(k-j)} \max _{L_{k, j}} D\left(L_{k, j}\right)
$$

Воспользуемся тем, что множество $D\left(L_{k, j}\right)$ определено по набору линейных соотношений $L_{k, j}$ точно так же, как множество $B\left(x^{1}, \ldots, x^{r}\right)$ выше было определено системой $L_{b}\left(x^{1}, \ldots, x^{r}\right)$. Поэтому их свойства во многом совпадают. В частности, наряду с леммой 4 справедливо (и доказывается теми же рассуждениями) следующее утверждение. 
Лемма 6. Пусть $2 \leqslant j \leqslant k-1$. Тогда

$$
\left|D\left(L_{k, j}\right)\right| \leqslant \rho(D)|D|^{j} .
$$

Используя (34) и (35), получаем, что

$$
\left|\bar{D}_{k, j}\right| \leqslant q^{k(k-j)} \rho(D)|D|^{j} .
$$

Значит, из соотношения $\rho(D) \rightarrow 0$ следует, что $\left|\bar{D}_{k, j}\right| /|D|^{j} \rightarrow 0$. Лемма 5 доказана.

Доказательство теоремы 2. Заметим, что условия теоремы 4 (с точностью до замены условия (9) условием (16)) повторяют условия теоремы 2 применительно к подмножествам $R_{1}, \ldots, R_{s}$ множества $J \subseteq D \times B$. Возьмем $J=D \times B, R_{j}=D \times B_{j}, j=1, \ldots, s$, $B=B_{1} \cup \ldots \cup B_{s}$. Тогда $\lambda_{j}^{\prime}=\lambda_{j}, j=1, \ldots, s$. Используя теорему 4 и следствие 6 , получаем утверждение теоремы 2.

Доказательство теоремь 3. Напомним, что согласно (11)

$$
l_{r}(D, B)=\left|\left\{k \in\{1, \ldots, M\}:\left|D B_{k}\right|=r\right\}\right|, \quad r=1, \ldots, q-1 .
$$

где $D B_{1}, \ldots, D B_{M}-$ разбиение множества $D \times B$ на классы подобных векторов и $M-$ общее число таких классов. Выберем из каждого множества $D B_{k}$ по одному произвольному вектору $d b_{k} \in D B_{k}$ и положим $J=\left\{d b_{1}, d b_{2}, \ldots, d b_{M}\right\} \subseteq D \times B$. Построим разбиение этого множества на непересекающиеся множества $R_{1}, \ldots, R_{q-1}$ по правилу

$$
R_{k}=\left|\left\{d b_{k}: k \in\{1, \ldots, M\},\left|D B_{k}\right|=r\right\}\right|, \quad r=1, \ldots, q-1,
$$

и введем случайные величины

$$
\xi_{r}=\sum_{(x, b) \in R_{k}} I\{A x=b\}, \quad r=1, \ldots, q-1,
$$

с множествами $R_{1}, \ldots, R_{q-1}$ из (36). Тогда

$$
\xi(D, A, B)=\xi_{1}+2 \xi_{2}+\ldots+(q-1) \xi_{q-1} .
$$

Используя предположения теоремы 3 и следствие 6, нетрудно убедиться, что для случайных величин (37) выполнены условия теоремы 4, причем $\lambda_{j}^{\prime}=\lambda_{j}, j=1, \ldots, q-1$. Поэтому, согласно теореме 4 , распределение вектора $\left(\xi_{1}, \ldots, \xi_{q-1}\right)$ сходится к распределению вектора $\left(\pi_{1}, \ldots, \pi_{q-1}\right)$, компоненты которого определены в формулировке теоремы 3 . Осталось воспользоваться равенством (38). Теорема 3 доказана.

\section{5. Доказательство теоремы 1}

Размерность пространства в этом разделе обозначим символом $T$. Во всех предельных переходах считается, что $T \rightarrow \infty$. Будем также использовать обозначения

$$
\begin{aligned}
& S_{r}(d)=\left\{x=\left(x^{1}, \ldots, x^{T}\right) \in V^{T}: 1 \leqslant\|x-d\| \leqslant r\right\}, \\
& S_{r}^{\prime}(d)=\left\{x=\left(x^{1}, \ldots, x^{T}\right) \in V^{T}:\|x-d\|=r\right\} .
\end{aligned}
$$


Пусть $B$ - заданное подмножество $V^{T}, a_{1}, a_{2}, a_{3} \in K \backslash\{0\}, d \in V^{T}$ и $N\left(a_{1}, a_{2}, a_{3}, d, B\right)$ - число решений уравнения

$$
a_{1} u^{1} \oplus \ldots \oplus a_{3} u^{3}=d
$$

относительно тройки $\left(u^{1}, u^{2}, u^{3}\right) \in B^{3}$. Пусть $T \rightarrow \infty$. Нас интересуют условия на характер изменения множества $B$, при которых для величины

$$
\rho(B)=\frac{N(B)}{|B|^{2}},
$$

где

$$
N(B)=\max _{a_{1}, a_{2}, a_{3}, d} N\left(a_{1}, a_{2}, a_{3}, d, B\right),
$$

выполнено соотношение $\rho(B) \rightarrow 0$.

Пусть случайные векторы $\xi^{1}, \xi^{2}$ выбраны случайно и независимо из множества $B$ в соответствии с равномерным распределением на нем. Тогда

$$
N\left(a_{1}, a_{2}, a_{3}, d, B\right)=|B|^{2} \mathbf{P}\left\{a_{1} \xi^{1} \oplus a_{2} \xi^{2} \in a_{3} B \oplus d\right\},
$$

а условие $\rho(B) \rightarrow 0$ эквивалентно соотношению

$$
\max _{a_{1}, a_{2}, a_{3}, d} \mathbf{P}\left\{a_{1} \xi^{1} \oplus a_{2} \xi^{2} \in a_{3} B \oplus d\right\} \rightarrow 0 .
$$

Когда множество $B$ замкнуто относительно умножения на ненулевые элементы поля, это соотношение можно упростить, взяв в качестве множителей $a_{1}, a_{2}, a_{3}$ единицы. В частности, когда $B=S_{r}(0)$, условие (3) принимает вид

$$
\max _{d} \mathbf{P}\left\{\xi^{1} \oplus \xi^{2} \in S_{r}(d)\right\} \rightarrow 0 .
$$

Аналогичный вид это условие принимает в случае, когда $B=S_{r}^{\prime}(0)$.

Для доказательства теоремы 1 потребуется информация об асимптотических свойствах суммы случайных векторов $\xi^{1}$ и $\xi^{2}$, которую мы получим из следующей леммы.

Лемма 7. Пусть множество $B \in\left\{S_{r}(0), S_{r}^{\prime}(0)\right\}$, случайные векторы $\xi^{1}, \xi^{2} \ldots, \xi^{s}, s \geqslant 2$, выбраны случайно и независимо в соответствии с равномерным распределением на множестве В и для параметров $T, r$ выполнень условия

$$
T \rightarrow \infty, \quad r \rightarrow \infty, \quad r T^{-1} \leqslant \rho<(q-1) / q .
$$

Тогда при любом $\varepsilon>0$

$$
\begin{aligned}
& \mathbf{E}\left\|\xi^{1} \oplus \ldots \oplus \xi^{s}\right\|=r \sum_{k=0}^{s-1}\left(1-\frac{r}{T} \frac{q}{q-1}\right)^{k}\left(1+O\left(r^{\varepsilon-1}\right)\right), \\
& \mathbf{D}\left\|\xi^{1} \oplus \ldots \oplus \xi^{s}\right\|=O\left(r^{1+\varepsilon}\right) .
\end{aligned}
$$

В случае $S_{r}^{\prime}(0)$ множитель $1+O\left(r^{\varepsilon-1}\right)$ в соотношении (43) равен единице, а равенство (44) принимает вид

$$
\mathbf{D}\left\|\xi^{1} \oplus \ldots \oplus \xi^{s}\right\|=O(r) .
$$


Доказательство. Пусть $B=S_{r}(0)$. Положим

$$
\eta_{i}(s)=I\left\{\left(\xi^{1} \oplus \ldots \oplus \xi^{s}\right)_{i} \neq 0\right\}, \quad i=1, \ldots, T,
$$

где $(x)_{i}-i$-я координата вектора $x$. Тогда

$$
\mathbf{E}\left\|\xi^{1} \oplus \ldots \oplus \xi^{s}\right\|=\sum_{i=1}^{T} \mathbf{P}\left\{\eta_{i}(s)=1\right\} .
$$

Найдем величины $\mathbf{P}\left\{\eta_{i}(s)=1\right\}$. Введем обозначения

$$
p_{i}(\alpha \mid \beta)=\mathbf{P}\left\{\eta_{i}(s+1)=\alpha \mid \eta_{i}(s)=\beta\right\}, \quad \alpha, \beta \in\{0,1\} .
$$

Тогда при всех $s=1,2, \ldots$

$$
\begin{aligned}
\mathbf{P}\left\{\eta_{i}(s+1)=1\right\} & =\mathbf{P}\left\{\eta_{i}(s)=1\right\} p_{i}(1 \mid 1)+\mathbf{P}\left\{\eta_{i}(s)=0\right\} p_{i}(1 \mid 0) \\
& =\mathbf{P}\left\{\eta_{i}(s)=1\right\}\left(p_{i}(1 \mid 1)-p_{i}(1 \mid 0)\right)+p_{i}(1 \mid 0) .
\end{aligned}
$$

Используя рекуррентное соотношение (47), получаем для производящей функции

$$
\varphi(z)=\sum_{s=1}^{\infty} \mathbf{P}\left\{\eta_{i}(s)=1\right\} z^{s}
$$

равенство

$$
\varphi(z)\left(1-z\left(p_{i}(1 \mid 1)-p_{i}(1 \mid 0)\right)\right)=p_{i}(1 \mid 0) \sum_{s=1}^{\infty} z^{s}+z\left(\mathbf{P}\left\{\eta_{i}(1)=1\right\}-p_{i}(1 \mid 0)\right) .
$$

Согласно определениям,

$$
\left.\mathbf{P}\left\{\eta_{i}(1)=1\right\}=p_{i}(1 \mid 0)\right)
$$

Поэтому

$$
\left.\varphi(z)=p_{i}(1 \mid 0)\right) \sum_{s=1}^{\infty} z^{s} \sum_{s=0}^{\infty}\left(p_{i}(1 \mid 1)-p_{i}(1 \mid 0)\right)^{s} z^{s} .
$$

Разлагая это выражение в степенной ряд, получаем, что

$$
\left.\mathbf{P}\left\{\eta_{i}(s)=1\right\}=p_{i}(1 \mid 0)\right) \sum_{k=0}^{s-1}\left(p_{i}(1 \mid 1)-p_{i}(1 \mid 0)\right)^{k} .
$$

Осталось найти величины $\left.p_{i}(1 \mid \alpha)\right), \alpha \in\{0,1\}$. Согласно (46),

$$
\begin{aligned}
& p_{i}(1 \mid 1)=\mathbf{P}\left\{\xi_{i}^{s+1}=0\right\}+\mathbf{P}\left\{\xi_{i}^{s+1} \neq 0, \xi_{i}^{s+1} \neq-\left(\xi_{i}^{1}+\ldots+\xi_{i}^{s}\right)\right\}, \\
& p_{i}(1 \mid 0)=\mathbf{P}\left\{\xi_{i}^{s+1} \neq 0\right\} .
\end{aligned}
$$

Раскрывая слагаемые в правых частях равенств (49), получаем равенства

$$
\begin{aligned}
& p_{i}(1 \mid 1)=\frac{1}{\left|S_{r}(0)\right|} \sum_{w=1}^{r}\left(\begin{array}{l}
T \\
w
\end{array}\right)(q-1)^{w}\left(1-(q-1)^{-1} \frac{w}{T}\right), \\
& p_{i}(1 \mid 0)=\frac{1}{\left|S_{r}(0)\right|} \sum_{w=1}^{r}\left(\begin{array}{l}
T \\
w
\end{array}\right)(q-1)^{w} \frac{w}{T}=\mathbf{P}\left\{\eta_{i}(1)=1\right\} .
\end{aligned}
$$


Значит,

$$
p_{i}(1 \mid 1)-p_{i}(1 \mid 0)=\frac{1}{\left|S_{r}(0)\right|} \sum_{w=1}^{r}\left(\begin{array}{l}
T \\
w
\end{array}\right)(q-1)^{w}\left(1-\frac{q}{q-1} \frac{w}{T}\right)
$$

где

$$
\left|S_{r}(0)\right|=\sum_{w=1}^{r}\left(\begin{array}{l}
T \\
w
\end{array}\right)(q-1)^{w}
$$

Из (45), (48) и (51) следует, что

$$
\begin{aligned}
\mathbf{E}\left\|\xi^{1} \oplus \ldots \oplus \xi^{s}\right\| & =\frac{1}{\sum_{w=1}^{r}\left(\begin{array}{l}
T \\
w
\end{array}\right)(q-1)^{w}} \sum_{w=1}^{r}\left(\begin{array}{c}
T \\
w
\end{array}\right)(q-1)^{w} w \\
& \times \sum_{k=0}^{s-1}\left(\frac{1}{\sum_{w=1}^{r}\left(\begin{array}{c}
T \\
w
\end{array}\right)(q-1)^{w}} \sum_{w=1}^{r}\left(\begin{array}{c}
T \\
w
\end{array}\right)(q-1)^{w}\left(1-\frac{q}{q-1} \frac{w}{T}\right)\right)^{k} .
\end{aligned}
$$

Стандартными рассуждениями нетрудно показать, что при условии (42) и любых $m, \varepsilon>0$

$$
\frac{1}{\sum_{w=1}^{r}\left(\begin{array}{l}
T \\
w
\end{array}\right)(q-1)^{w}} \sum_{w=1}^{r}\left(\begin{array}{c}
T \\
w
\end{array}\right)(q-1)^{w} w^{m}=r^{m}\left(1+O\left(r^{\varepsilon-1}\right)\right) .
$$

Поэтому из (50) следует, что

$$
\begin{aligned}
& p_{i}(1 \mid 1)=\left(1-\frac{r}{T(q-1)}\right)\left(1+O\left(r^{\varepsilon-1}\right)\right), \\
& p_{i}(1 \mid 0)=\frac{r}{T}\left(1+O\left(r^{\varepsilon-1}\right)\right),
\end{aligned}
$$

а из (52) следует (43).

Оценим дисперсию $\mathbf{D}\left\|\xi^{1} \oplus \ldots \oplus \xi^{s}\right\|$. Введем обозначения

$$
\begin{aligned}
P_{i}^{(\alpha)}(s) & =\mathbf{P}\left\{\eta_{i}(s)=\alpha\right\}, \\
P_{i, j}^{(\alpha, \beta)}(s) & =\mathbf{P}\left\{\eta_{i}(s)=\alpha, \eta_{j}(s)=\beta\right\}
\end{aligned}
$$

и запишем равенство

$$
\begin{aligned}
\mathbf{D}\left\|\xi^{1} \oplus \ldots \oplus \xi^{s}\right\| & =\mathbf{D}\left(\eta_{1}(s)+\ldots+\eta_{T}(s)\right) \\
& =\sum_{i=1}^{T} P_{i}^{(1)}(s)\left(1-P_{i}^{(1)}(s)\right)+2 \sum_{1 \leqslant i<j \leqslant T}\left(P_{i, j}^{(1,1)}(s)-P_{i}^{(1)}(s) P_{j}^{(1)}(s)\right) .
\end{aligned}
$$

Задача сводится к вычислению величин $P_{i, j}^{(1,1)}(s)$. Для этого используем соотношения

$$
\begin{aligned}
P_{i, j}^{(1,1)}(s+1) & =\sum_{\alpha, \beta \in\{0,1\}} P_{i, j}^{(\alpha, \beta)}(s) p_{i, j}(1,1 \mid \alpha, \beta), \quad s=1,2, \ldots, \\
p_{i, j}(1,1 \mid \alpha, \beta) & =\mathbf{P}\left\{\eta_{i}(s+1)=1, \eta_{j}(s+1)=1 \mid \eta_{i}(s)=\alpha, \eta_{j}(s)=\beta\right\} .
\end{aligned}
$$


Вычислим сначала величины $P_{i, j}^{(\alpha, \beta)}(1)$ и $p_{i, j}(1,1 \mid \alpha, \beta)$. Справедливо равенство

$$
\begin{aligned}
& P_{i, j}^{(\alpha, \beta)}(1)=\alpha \beta \mathbf{P}\left\{\xi_{i}^{1} \neq 0, \xi_{j}^{1} \neq 0\right\}+(1-\alpha) \beta \mathbf{P}\left\{\xi_{i}^{1}=0, \xi_{j}^{1} \neq 0\right\} \\
& +\alpha(1-\beta) \mathbf{P}\left\{\xi_{i}^{1} \neq 0, \xi_{j}^{1}=0\right\}+(1-\alpha)(1-\beta) \mathbf{P}\left\{\xi_{i}^{1}=0, \xi_{j}^{1}=0\right\} .
\end{aligned}
$$

Следовательно,

$$
\begin{aligned}
P_{i, j}^{(\alpha, \beta)}(1)\left|S_{r}(0)\right|=\alpha & \beta \sum_{w=2}^{r}\left(\begin{array}{c}
T-2 \\
w-2
\end{array}\right)(q-1)^{w}+(\alpha+\beta-2 \alpha \beta) \sum_{w=1}^{r}\left(\begin{array}{c}
T-2 \\
w-1
\end{array}\right)(q-1)^{w} \\
& +(1-\alpha)(1-\beta) \sum_{w=1}^{r}\left(\begin{array}{c}
T-2 \\
w
\end{array}\right)(q-1)^{w} \\
=\alpha \beta & \sum_{w=2}^{r}\left(\begin{array}{l}
T \\
w
\end{array}\right)(q-1)^{w} \frac{w(w-1)}{T(T-1)} \\
& +(\alpha+\beta-2 \alpha \beta) \sum_{w=1}^{r}\left(\begin{array}{c}
T \\
w
\end{array}\right)(q-1)^{w} \frac{w(T-w)}{T(T-1)} \\
& +(1-\alpha)(1-\beta) \sum_{w=1}^{r}\left(\begin{array}{c}
T \\
w
\end{array}\right)(q-1)^{w} \frac{(T-w)(T-w-1)}{T(T-1)} .
\end{aligned}
$$

Отсюда и из (53) получаем, что

$$
\begin{aligned}
& \frac{P_{i, j}^{(\alpha, \beta)}(1)}{1+O\left(r^{\varepsilon-1}\right)}=\alpha \beta\left(\frac{r}{T}\right)^{2}+(\alpha+\beta-2 \alpha \beta) \frac{r}{T}\left(1-\frac{r}{T}\right)+(1-\alpha)(1-\beta)\left(1-\frac{r}{T}\right)^{2}, \\
& \frac{P_{i}^{(\alpha)}(1)}{1+O\left(r^{\varepsilon-1}\right)}=\alpha \frac{r}{T}+(1-\alpha)\left(1-\frac{r}{T}\right) .
\end{aligned}
$$

Поэтому

$$
P_{i, j}^{(\alpha, \beta)}(1)=P_{i}^{(\alpha)}(1) P_{j}^{(\beta)}(1)\left(1+O\left(r^{\varepsilon-1}\right)\right) .
$$

Аналогично доказывается, что при $\alpha, \beta \in\{0,1\}$

$$
p_{i, j}(1,1 \mid \alpha, \beta)=p_{i}(1 \mid \alpha) p_{j}(1 \mid \beta)\left(1+O\left(r^{\varepsilon-1}\right)\right) .
$$

Действительно (здесь мы опускаем промежуточные выкладки),

$$
\begin{aligned}
& p_{i, j}(1,1 \mid \alpha, \beta)\left|S_{r}(0)\right| \\
& =\alpha \beta \sum_{w=1}^{r}\left(\left(\begin{array}{c}
T-2 \\
w
\end{array}\right)(q-1)^{w}+2\left(\begin{array}{c}
T-2 \\
w-1
\end{array}\right)(q-2)(q-1)^{w-1}+\left(\begin{array}{c}
T-2 \\
w-2
\end{array}\right)(q-2)^{2}(q-1)^{w-2}\right) \\
& \quad+(\alpha+\beta-2 \alpha \beta) \sum_{w=1}^{r}\left(\left(\begin{array}{c}
T-2 \\
w-1
\end{array}\right)(q-1)^{w}+\left(\begin{array}{c}
T-2 \\
w-2
\end{array}\right)(q-2)(q-1)^{w-1}\right) \\
& \quad+(1-\alpha)(1-\beta) \sum_{w=1}^{r}\left(\begin{array}{c}
T-2 \\
w-2
\end{array}\right)(q-1)^{w},
\end{aligned}
$$


где полагаем $\left(\begin{array}{l}n \\ v\end{array}\right)=0$ при $v<0$. Воспользуемся теперь $(53)$ и получим равенство

$$
\begin{aligned}
\frac{p_{i, j}(1,1 \mid \alpha, \beta)}{1+O\left(r^{\varepsilon-1}\right)}=\alpha & \beta\left(1-\frac{r}{(q-1) T}\right)^{2} \\
& +(\alpha+\beta-2 \alpha \beta) \frac{r}{T}\left(1-\frac{r}{(q-1) T}\right)+(1-\alpha)(1-\beta)\left(\frac{r}{T}\right)^{2} .
\end{aligned}
$$

Из (61) и (54) получаем (59). Теперь, используя рекуррентную формулу (56) и соотношения (58), (59), индукцией по значениям $s$ выводим оценки

$$
P_{i, j}^{(1,1)}(s)=P_{i}^{(1)}(s) P_{j}^{(1)}(s)\left(1+O\left(r^{\varepsilon-1}\right)\right), \quad s=1,2, \ldots,
$$

равномерные по $1 \leqslant i<j \leqslant T$. Из (55) и (62) с учетом соотношений

$$
\sum_{i=1}^{T} P_{i}^{(1)}(s)=\mathbf{E}\left\|\xi^{1} \oplus \ldots \oplus \xi^{s}\right\|=O(r)
$$

получаем оценку (44).

Если $B=S_{r}^{\prime}(0)$, то доказательство соотношений (43) и (44) повторяет (с очевидными упрощениями) доказательство для случая, когда $B=S_{r}(0)$. При этом множитель $1+O\left(r^{\varepsilon-1}\right)$ в (43) заменяется единицей, а для дисперсии вместо (44) получается оценка

$$
\mathbf{D}\left\|\xi^{1} \oplus \ldots \oplus \xi^{s}\right\|=O(r)
$$

Лемма 7 доказана.

Доказательство теоремы 1. Проверим свойство (3) для множества $B=S_{r}(0)$. Пусть $\xi^{1}$ и $\xi^{2}-$ независимые случайные векторы, распределенные на множестве $S_{r}(0)$ равномерно. Так как их распределения инвариантны относительно перестановки и умножения координат на ненулевые элементы поля, то этими же свойствами обладает и их сумма $\xi^{1} \oplus \xi^{2}$. Поэтому при $\|x\|=k, k=0,1, \ldots$,

$$
\mathbf{P}\left\{\xi^{1} \oplus \xi^{2}=x\right\}=\frac{1}{(q-1)^{k}\left(\begin{array}{c}
T \\
k
\end{array}\right)} \mathbf{P}\left\{\left\|\xi^{1} \oplus \xi^{2}\right\|=k\right\} .
$$

Сначала проверим выполнение условия (41) при условии (42), то есть $T, r \rightarrow \infty$, $r T^{-1} \leqslant \rho<(q-1) / q$, а затем рассмотрим случай, когда $T \rightarrow \infty, 1 \leqslant r=O(1)$. Согласно лемме 7 , при любом $\varepsilon>0$

$$
\begin{aligned}
& \mathbf{E}\left\|\xi^{1} \oplus \xi^{2}\right\|=r_{2}\left(1+O\left(r^{\varepsilon-1}\right)\right), \\
& \mathbf{D}\left\|\xi^{1} \oplus \xi^{2}\right\|=O\left(r^{1+\varepsilon}\right),
\end{aligned}
$$

где

$$
r_{2}=r+r\left(1-\frac{r}{T} \frac{q}{q-1}\right) .
$$

Исходя из (65) и неравенств $r T^{-1} \leqslant \rho<(q-1) / q$, выберем такое $0<\varepsilon<1$, что

$$
r(1+\varepsilon)<r_{2}(1-\varepsilon), \quad r_{2}(1+\varepsilon)<T(q-1) / q .
$$


Используя (63), получаем, что

$$
\begin{aligned}
\mathbf{P}\left\{\xi^{1} \oplus \xi^{2} \in S_{r}(d)\right\}= & \mathbf{P}\left\{\xi^{1} \oplus \xi^{2} \in S_{r}(d),\left\|\xi^{1} \oplus \xi^{2}\right\| \leqslant r_{2}(1-\varepsilon)\right\} \\
& +\mathbf{P}\left\{\xi^{1} \oplus \xi^{2} \in S_{r}(d),\left\|\xi^{1} \oplus \xi^{2}\right\| \geqslant r_{2}(1+\varepsilon)\right\} \\
& +\mathbf{P}\left\{\xi^{1} \oplus \xi^{2} \in S_{r}(d), r_{2}(1-\varepsilon)<\left\|\xi^{1} \oplus \xi^{2}\right\|<r_{2}(1+\varepsilon)\right\} \\
\leqslant & \mathbf{P}\left\{\left\|\xi^{1} \oplus \xi^{2}\right\| \leqslant r_{2}(1-\varepsilon)\right\}+\mathbf{P}\left\{\left\|\xi^{1} \oplus \xi^{2}\right\|\right. \\
\leqslant & \left.r_{2}(1+\varepsilon)\right\}+\sum_{r_{2}(1-\varepsilon)<k<r_{2}(1+\varepsilon)} \frac{\left|S_{r}(d)\right|}{(q-1)^{k}\left(\begin{array}{l}
T \\
k
\end{array}\right)} \mathbf{P}\left\{\left\|\xi^{1} \oplus \xi^{2}\right\|=k\right\} .
\end{aligned}
$$

Из соотношений (64) и неравенства Чебышёва следует, что первое и второе слагаемые в правой части неравенства (67) стремятся к нулю. В силу правого неравенства (66), в интервале $r_{2}(1-\varepsilon)<k<r_{2}(1+\varepsilon)$ величина $(q-1)^{k}\left(\begin{array}{l}T \\ k\end{array}\right)$ возрастает вместе с $k$ (эта величина имеет точку максимума $k^{*}, k^{*} / T \rightarrow(q-1) / q$ при $\left.T \rightarrow \infty\right)$. Кроме того,

$$
\left|S_{r}(d)\right|<r(q-1)^{r}\left(\begin{array}{l}
T \\
r
\end{array}\right)
$$

Поэтому

$$
\begin{aligned}
& \sum_{r_{2}(1-\varepsilon)<k<r_{2}(1+\varepsilon)} \frac{\left|S_{r}(d)\right|}{(q-1)^{k}\left(\begin{array}{l}
T \\
k
\end{array}\right)} \mathbf{P}\left\{\left\|\xi^{1} \oplus \xi^{2}\right\|=k\right\} \leqslant \frac{\left|S_{r}(d)\right|}{(q-1)^{\left[r_{2}(1-\varepsilon)\right]\left(\begin{array}{c}
T \\
{\left[r_{2}(1-\varepsilon)\right]}
\end{array}\right)}}
\end{aligned}
$$

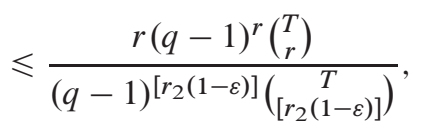

где $[a]$ - целая часть числа $a$. В силу левого неравенства в (66), последняя дробь в (68) стремится к нулю.

Таким образом,

$$
\mathbf{P}\left\{\xi^{1} \oplus \xi^{2} \in S_{r}(d)\right\} \rightarrow 0
$$

при $T \rightarrow \infty, r \rightarrow \infty, r T^{-1} \leqslant \rho<(q-1) / q$ равномерно по $d \in V^{T}$. Осталось отметить, что при $T \rightarrow \infty, 1 \leqslant r=O(1)$, выполнено соотношение

$$
\mathbf{P}\left\{\left\|\xi^{1} \oplus \xi^{2}\right\|=2 r\right\} \rightarrow 1,
$$

поэтому

$$
\mathbf{P}\left\{\xi^{1} \oplus \xi^{2} \in S_{r}(d)\right\} \leqslant \mathbf{P}\left\{\left\|\xi^{1} \oplus \xi^{2}\right\| \neq 2 r\right\}+\mathbf{P}\left\{\left\|\xi^{1} \oplus \xi^{2}\right\|=2 r\right\} \frac{\left|S_{r}(d)\right|}{(q-1)^{2 r}\left(\begin{array}{c}
T \\
2 r
\end{array}\right)} \rightarrow 0 .
$$

Значит, в условиях теоремы 1 свойства (41) и $\rho\left(S_{r}(0)\right) \rightarrow 0$ выполнены. Тогда (см. замечание 1$)$ и $\rho\left(S_{r}\left(b^{0}\right)\right) \rightarrow 0$.

Доказательство теоремы в случае, когда $B=S_{r}^{\prime}\left(b^{0}\right)$ повторяет предшествующие рассуждения с заменой во всех выкладках множества $S_{r}(d)$ множеством $S_{r}^{\prime}(d)$ и заменой в правой части последнего неравенства (68) множителя $r$ единицей. Теорема 1 доказана.

Авторы благодарны А. М. Зубкову и Б. А. Севастьянову за полезные замечания. 


\section{Список литературы}

1. Копытцев В. А., О числе решений систем линейных булевых уравнений в множестве векторов, обладающих заданным числом единиц. Дискретная математика (2002) 14, №4, 87-109.

2. Копытцев В. А., О числе решений системы случайных линейных уравнений. Дискретная математика (2006) 18, №1, 40-62.

3. Михайлов В. Г., О предельной теореме Б. А. Севастьянова для сумм зависимых случайных индикаторов. Обозрение прикладной и промышленной математики (2003) 10, №3, 571-578.

4. Михайлов В. Г., Предельные теоремы для числа точек случайного линейного подпространства, попавших в заданное множество. Дискретная математика (2003) 15, №2, 128-137.

5. Михайлов В. Г., Предельные теоремы для числа решений системы случайных линейных уравнений, попавших в заданное множество. Дискретная математика (2007) 19, №1, 17-26.

Статья поступила 11.03.2010. 